\title{
Synthesis of Hoodigogenin A, the Aglycone of Hoodigosides Extracted from Hoodia gordonii
}

\section{Philippe Geoffroy, Blandine Ressault and Michel Miesch*}

Department of Chemistry, University of Strasbourg, France

\section{Introduction}

Hoodia gordonii is a succulent plant (asclepiadaceae family) which grows in the Kalahari desert in South Africa. On a historical point of view, it was claimed that the San people, a Bushmen tribe leaving in the Kalahari desert, were able to make long hunting trips without feeling thursty and hungry after chewing fresh sap from $H$. gordonii. Therefore, it was claimed that $H$. gordonii could represent a new help for fighting obesity, which is one of the major health problems in the 21st century. Indeed, in 2014, more than 1.9 billion adults, 18 years and older, were overweight. Of these over 600 million were obese [1].

In 2007, Van Herdeen et al. reported for the first time the appetite suppressant properties of $\mathrm{H}$. gordonii [2,3]. According to these authors, the compound responsible for that, is the preganane glycoside P57AS3. Later on, Shukla et al. reported that more than 40 different pregnane glycosides (Hoodigosides) were isolated from H. gordonii [4,5]. The common aglycone of all these compounds is Hoodigogenin A (or Gordonoside A) (Figure 1).

The yield of extraction of the Hoodigosides from Hoodia gordonii lies between $0.003 \%$ and $0.02 \%$. On the other hand, the isolation of analytically pure compounds proved to be highly time consuming and tedious. Therefore, in the frame of a collaborative study concerning the synthesis, the extraction and the biological evaluation of Hoodigogenin A, we developed an original synthesis of Hoodigogenin A starting from commercially available reagents. Indeed, Hoodigogenin A could provide different Hoodigosides after running a glycosylation reaction with the required sugar moiety. We report herein the synthesis of Hoodigogenin A, the key step being a Norrish type I-Prins reaction.

\section{Synthesis of Hoodigogenin A}

The synthesis started from the commercially available compound 1. After a regioselective deprotection of the $3 \alpha$-acetoxy group and oxidation of the resulting hydroxy group, a regioselective $\alpha$-bromination of the diketo derivative 2 yielded stereospecifically the $\beta$-bromoketo derivative 3. A dehydrobromination reaction led to the $\alpha, \beta$-unsaturated keto derivative 4 [6]. The latter was treated with acetic anhydride and acetyl chloride to give readily the dienol acetate 5 [7]. After protection of the carbonyl group with ethyleneglycol, the dienol acetate was reduced in the presence of $\mathrm{NaBH} 4[8,9]$ followed by a $\mathrm{KOH}$ promoted deprotection of the 12 a-acetate group, allowing the introduction of the double bond in the B ring and yielding the unstable diol 6 . A regioselective protection of the $3 \beta$-hydroxyl group gave compound 7, which was subjected to a Dess Martin periodane oxidation [10] to afford the keto derivative 8 (Figure 2).

Compound $\mathbf{8}$ was then suitable to undergo a Norrish type I reaction [11-15]. It has to be noted that the photochemical ring opening of pregnenolone derivatives was never reported in the literature. We were pleased to see that the photolysis of compound $\mathbf{8}$, which was carried out in a quartz apparatus with a $125 \mathrm{~W}$ high pressure mercury lamp, led readily to the formation of aldehyde $\mathbf{9}$. The latter proved to be instable. Therefore the subsequent Prins reaction was directly carried out on the crude photolysis reaction mixture. Under these reactions conditions, three compounds were isolated: compound $\mathbf{1 0}$ that resulted from a deprotection of the dioxolane 8 , the spiro derivative 11 and the desired compound 12, which was isolated in $25 \%$ yield (Figure 3) [16].

Finally, the protection of the $12 \beta$-hydroxy group with tigloyl chloride in the presence of pyridine and DMAP afforded compound 13. Deprotection of the $3 \beta$-acetate group gave Hoodigogenin A, whose structure was confirmed by ${ }^{1} \mathrm{H}$ and ${ }^{13} \mathrm{C}$ NMR analysis and by X-ray analysis (Figures 4 and 5) [17].

\section{Conclusion}

To overcome the limited availability of Hoodigogenin A, we have disclosed an original synthetic route that affords the latter in $3 \%$ overall yield. This synthesis represents a very interesting alternative to the extraction methods for which the yields are 100 times lower. Moreover, analogs of Hoodigogenin A are now accessible by our method [18]. Concerning the biological activities, Smith et al. reported recently a summary of the current knowledge concerning the efficiency of $H$. gordonii as an appetite suppressant drug. Many contradictory results were obtained and it clearly came out that more studies are absolutely necessary to elucidate the mode of action of hoodigosides [19].

\section{Experimental section}

Melting points were measured on a Stuart Scientific melting point apparatus (SMP 3) and are uncorrected. Reactions were carried out under argon with magnetic stirring and degassed solvents. $\mathrm{Et}_{2} \mathrm{O}$ and THF were distilled from $\mathrm{Na}$ /benzophenone. Thin layer chromatography (TLC) was carried out on silica gel plates (Merck 60F254) and the spots were visualized under UV lamp (254 or $365 \mathrm{~nm}$ ) and sprayed with phosphomolybdic acid solution ( $25 \mathrm{~g}$ phosphomolybdic acid, 10 g cerium sulfate, $60 \mathrm{~mL} \mathrm{H}_{2} \mathrm{SO}_{4}, 940 \mathrm{~mL} \mathrm{H}_{2} \mathrm{O}$ ) followed by heating on a hot plate. For column chromatography, silica gel (Merck Si $6040-$ $60 \mu \mathrm{m}$ ) was used. IR spectra were recorded on Bruker Alpha (ATR) spectrophotomer. ${ }^{1} \mathrm{H}$ NMR spectra were recorded at $300 \mathrm{MHz}$ (Bruker AC-300) and ${ }^{13} \mathrm{C}$ NMR spectra at $75 \mathrm{MHz}$ (Bruker AC-300) using the signal of the residual nondeuterated solvent as internal reference. Significant ${ }^{1} \mathrm{H}$ NMR data are tabulated in the following order: chemical shift $(\delta)$ expressed in ppm, 6 multiplicity (s, singlet; $d$, doublet; $t$, triplet; q, quartet; m, multiplet), coupling constants in hertz, number

*Corresponding author: Michel Miesch, University of Strasbourg, Chemistry Institute, UMR, 7177 Synthetic Organic Chemistry Laboratory-1, rue Blaise Pascal, BP 296/R8, 67008 Strasbourg Cedex, France, Tel: 368851752; Fax: 368851754; E-mail:m.miesch@unistra.fr

Received October 16, 2015; Accepted December 03, 2015; Published December 07, 2015

Citation: Geoffroy P, Ressault B, Miesch M (2015) Synthesis of Hoodigogenin A the Aglycone of Hoodigosides Extracted from Hoodia gordonii. J Steroids Horm Sci 6: 163. doi:10.4172/2157-7536.1000163

Copyright: (c) 2015 Geoffroy P, et al.. This is an open-access article distributed under the terms of the Creative Commons Attribution License, which permits unrestricted use, distribution, and reproduction in any medium, provided the original author and source are credited. 


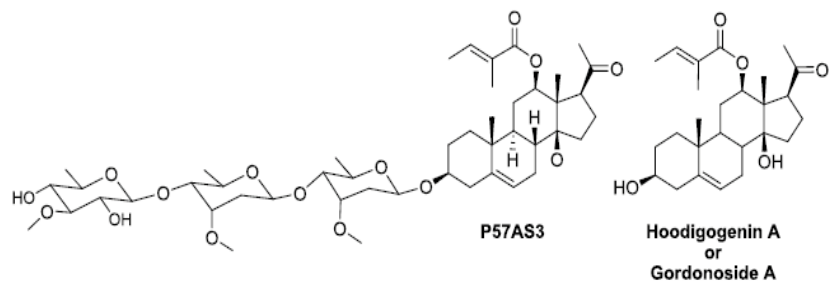

Figure 1: Pregnane glycoside extracted from $H$. gordonii and its aglycone 2.

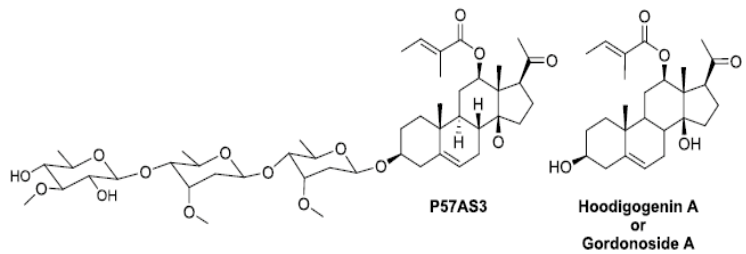

Figure 2: Reagents and reaction conditions: (a) i: $\mathrm{K}_{2} \mathrm{CO}_{3}, \mathrm{MeOH}, 25^{\circ} \mathrm{C} 1 \mathrm{~h}$ (83\%, (formation of compound $1 \mathrm{a})$; ii: $\mathrm{CrO}_{3}$, acetone, $25^{\circ} \mathrm{C}, 1 \mathrm{~h}$ (quant.); (b): $\mathrm{Br}_{2}, \mathrm{AcOH}, 25^{\circ} \mathrm{C}, 1.5 \mathrm{~h}$; (c): $\mathrm{LiCl}, \mathrm{DMF}$, reflux, $1 \mathrm{~h}$ (67\% for the 2 steps); (d) $\mathrm{AcCl}, \mathrm{Ac} 2 \mathrm{O}$, reflux $50^{\circ} \mathrm{C}, 1 \mathrm{~h}(85 \%)$; (e): i: ethylene glycol, toluene, reflux, $3 \mathrm{~h}$ (quant.); ii: $\mathrm{NaBH}_{4}, \mathrm{MeOH} / \mathrm{THF}(1 / 1), 25^{\circ} \mathrm{C}, 1.5 \mathrm{~h}$; iii: $\mathrm{KOH}, \mathrm{MeOH}$, reflux, $1 \mathrm{~h}$ (ii+iii: $60 \%$ ); (f): Ac2O, pyridine, DMAP, $\mathrm{CH}_{2} \mathrm{Cl}_{2}, 25^{\circ} \mathrm{C}, 2.5 \mathrm{~h}(80 \%)$; (g): DessMartin reagent, $\mathrm{CH}_{2} \mathrm{Cl}_{2}, 0^{\circ} \mathrm{C}->25^{\circ} \mathrm{C}, 1 \mathrm{~h}(99 \%)$

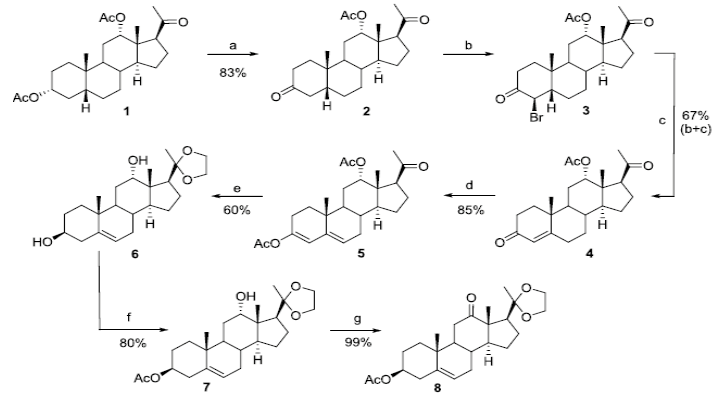

Figure 3: Reagents and reaction conditions: (a): $\mathrm{hv}, \mathrm{CH}_{2} \mathrm{Cl}_{2}, 25^{\circ} \mathrm{C}, 30 \mathrm{~min}$ (quant.); (b) $\mathrm{AcOH} / \mathrm{H}_{2} \mathrm{O} / \mathrm{TFA}$ (2.5/1/0.6), THF, $25^{\circ} \mathrm{C}$, $1.5 \mathrm{~h}$ [10 (19\%), 11 (15\%), $12(25 \%)]$.

of protons. High-resolution mass spectra (HRMS) were performed on a Agilent 6520 Accurate Mass Q-TOF.

\section{3a-hydroxy, 12a-acetoxy-5H $\beta$-pregnan-20-one (1a)}

To a solution of compound 1 (3.37 g, $8.05 \mathrm{mmol})$ in $\mathrm{MeOH}(80$ $\mathrm{mL}$ ) was added $\mathrm{K}_{2} \mathrm{CO}_{3}(1.00 \mathrm{~g}, 7.24 \mathrm{mmol})$. The reaction mixture was stirred for $1 \mathrm{~h}$ at r.t. and treated with water $(50 \mathrm{~mL})$. The aqueous layer was extracted with $\mathrm{Et}_{2} \mathrm{O}(3 \times 60 \mathrm{~mL})$. The combined organic layers were washed with brine $(1 \times 50 \mathrm{~mL})$, dried over $\mathrm{Na}_{2} \mathrm{SO}_{4}$, filtered and concentrated under reduced pressure. The white residue was purified by column chromatography (30 g silica gel, petroleum ether-ethyl acetate, 3:7), to afford compound $1 \mathrm{a}$ as a white solid (2.48 $\mathrm{g}, 83 \%, \mathrm{mp}$ 201.5-203.8 $\left.{ }^{\circ} \mathrm{C}\right)$. IR $\left(\mathrm{ATR}, \mathrm{cm}^{-1}\right): 3472,1714,1696 .{ }^{1} \mathrm{H} \mathrm{NMR}\left(\mathrm{CDCl}_{3}\right) \delta$ : $5.12\left(\mathrm{t},{ }^{1} \mathrm{H}, \mathrm{J}=2.7 \mathrm{~Hz}, \mathrm{H}-12 \beta\right) ; 3.63\left(\mathrm{tt},{ }^{1} \mathrm{H}, \mathrm{J}=10.9-4.6 \mathrm{~Hz}, \mathrm{H}-3 \beta\right) ; 2.95$ (t, $\left.{ }^{1} \mathrm{H}, \mathrm{J}=9.3 \mathrm{~Hz}, \mathrm{H}-17 \alpha\right) ; 2.20-2.05\left({ }^{1} \mathrm{H}, \mathrm{m}\right) ; 2.14$ (s, 3H, H-21); 2.01

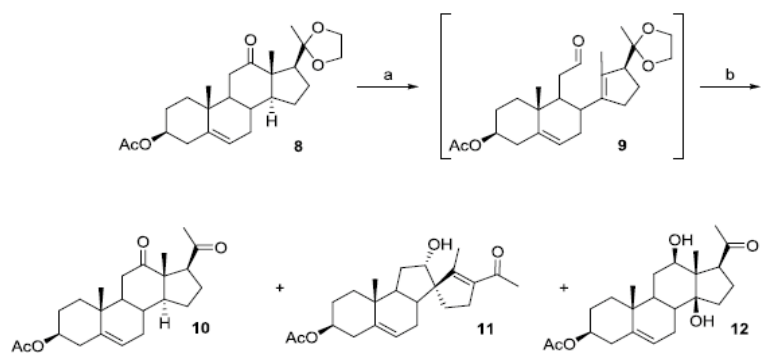

Figure 4: Reagents and reaction conditions: (a) tigloyl chloride, pyridine, DMAP, $\mathrm{CH}_{2} \mathrm{Cl}_{2}$, reflux, $7 \mathrm{~h}(80 \%)$; (b) $\mathrm{K}_{2} \mathrm{CO}_{3}, \mathrm{MeOH}, 25^{\circ} \mathrm{C}, 2 \mathrm{~h}(69 \%)$.

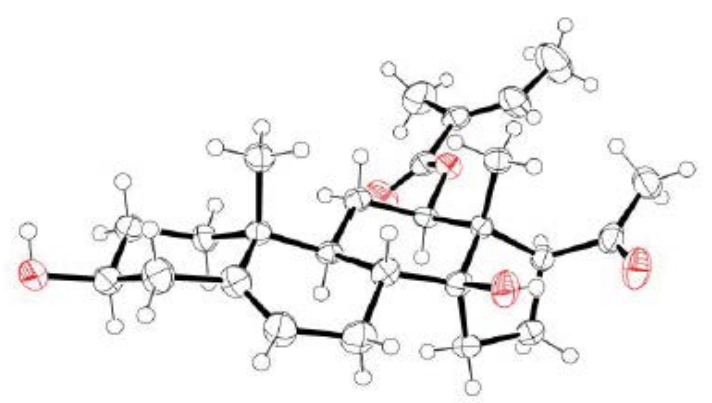

Figure 5: ORTEP drawing of Hoodigogenin A with thermal ellipsoids at the $30 \%$ probability level.

(s, 3H, $\left.\mathrm{CH}_{3}, \mathrm{Ac}\right) ; 1.95-0.85(\mathrm{~m}, 20 \mathrm{H}) ; 0.89\left(\mathrm{~s}, 3 \mathrm{H}, \mathrm{CH}_{3}\right) ; 0.67(3 \mathrm{H}, \mathrm{s}$, $\left.\mathrm{CH}_{3}\right) .{ }^{13} \mathrm{C} \mathrm{NMR}\left(\mathrm{CDCl}_{3}\right) \delta: 208.9(\mathrm{C}=\mathrm{O}, \mathrm{C}-20) ; 170.5(\mathrm{C}=\mathrm{O}, \mathrm{OAc}) ; 74.5$ (CH, C-3); $71.6(\mathrm{CH}, \mathrm{C}-12) ; 55.6(\mathrm{CH}) ; 49.6(\mathrm{CH}) ; 46.7(\mathrm{C}) ; 41.9(\mathrm{CH})$; $36.2\left(\mathrm{CH}_{2}\right) ; 35.6(\mathrm{CH}) ; 35.0\left(\mathrm{CH}_{2}\right) ; 34.5(\mathrm{CH}) ; 34.1(\mathrm{C}) ; 31.1\left(\mathrm{CH}_{3}\right) ; 30.4$ $(\mathrm{CH} 2) ; 27.0\left(\mathrm{CH}_{2}\right) ; 26.0\left(\mathrm{CH}_{2}\right) ; 25.6\left(\mathrm{CH}_{2}\right) ; 23.7\left(\mathrm{CH}_{2}\right) ; 23.0\left(\mathrm{CH}_{3}\right) ; 22.3$ $\left(\mathrm{CH}_{2}\right) ; 21.4\left(\mathrm{CH}_{3}\right) ; 13.8\left(\mathrm{CH}_{3}\right)$. HRMS (ESI) m/z: $\mathrm{C}_{23} \mathrm{H}_{36} \mathrm{NaO}_{4}[\mathrm{M}+\mathrm{Na}]+$ calcd. 399.2506, found 399.2518; [a]20D: +154 (c 0.01, $\left.\mathrm{CHCl}_{3}\right)$.

\section{$12 \alpha$-acetoxy-5H $\beta$-pregnan-3,20-dione (2)}

A solution of compound $1 \mathrm{a}(1.00 \mathrm{~g}, 2.66 \mathrm{mmol})$ in acetone $(60 \mathrm{~mL})$ at $0^{\circ} \mathrm{C}$ was treated dropwise with Jones reagent $(2.7 \mathrm{~mL})$. The orange solution was stirred for $1 \mathrm{~h}$ at r.t. and treated with water $(50 \mathrm{~mL})$. The aqueous layer was extracted with $\mathrm{Et}_{2} \mathrm{O}(3 \times 50 \mathrm{~mL})$. The combined organic layers were washed with sat. $\mathrm{NaHCO}_{3}(1 \times 50 \mathrm{~mL})$ and then with brine $(1 \times 50 \mathrm{~mL})$, dried over $\mathrm{Na}_{2} \mathrm{SO}_{4}$, filtered and concentrated under reduced pressure to afford compound 2 as a white solid (1.034 g, quant., mp $124-125.7^{\circ} \mathrm{C}$ ) which was used directly for the next step. IR (ATR, $\left.\mathrm{cm}^{-1}\right)$ : 1714-1699. ${ }^{1} \mathrm{H}$ NMR $\left(\mathrm{CDCl}_{3}\right) \delta: 5.16\left(\mathrm{t},{ }^{1} \mathrm{H}, \mathrm{J}=2.7 \mathrm{~Hz}\right.$, $\mathrm{H}-12 \beta) ; 2.93$ (t, $\left.{ }^{1} \mathrm{H}, \mathrm{J}=9 \mathrm{~Hz}, \mathrm{H}-17 \alpha\right) ; 2.63$ (dd, $\left.{ }^{1} \mathrm{H}, \mathrm{J}=13.5-15.3 \mathrm{~Hz}\right) ; 2.30-$ 1.05 (m, 19H); 2.11 (s, 3H, CH, $\mathrm{H}-21) ; 2.00\left(\mathrm{~s}, 3 \mathrm{H}, \mathrm{CH}_{3}, \mathrm{Ac}\right) ; 0.98(3 \mathrm{H}$, $\left.\mathrm{s}, \mathrm{CH}_{3}\right) ; 0.70\left(3 \mathrm{H}, \mathrm{s}, \mathrm{CH}_{3}\right) .{ }^{13} \mathrm{C} \mathrm{NMR}\left(\mathrm{CDCl}_{3}\right) \delta: 212.6(\mathrm{C}=\mathrm{O}, \mathrm{C}-3) ; 208.6$ (C=O, C-20); $170.3(\mathrm{C}=\mathrm{O}, \mathrm{OAc}) ; 74.3(\mathrm{CH}, \mathrm{C}-12) ; 55.5(\mathrm{CH}) ; 49.5(\mathrm{CH})$; $46.7(\mathrm{C}) ; 43.8(\mathrm{CH}) ; 42.1\left(\mathrm{CH}_{2}\right) ; 36.8\left(\mathrm{CH}_{2}\right) ; 36.5\left(\mathrm{CH}_{2}\right) ; 35.3(\mathrm{CH}) ; 34.7$ $(\mathrm{CH}) ; 34.3(\mathrm{C}) ; 31.1\left(\mathrm{CH}_{3}\right) ; 26.3\left(\mathrm{CH}_{2}\right) ; 25.9\left(\mathrm{CH}_{2}\right) ; 25.7\left(\mathrm{CH}_{2}\right) ; 23.6$ $\left(\mathrm{CH}_{2}\right) ; 22.3\left(\mathrm{CH}_{2}\right) ; 22.3\left(\mathrm{CH}_{3}\right) ; 21.3\left(\mathrm{CH}_{3}\right) ; 13.9(\mathrm{CH} 3)$. HRMS (ESI) $\mathrm{m} / \mathrm{z}: \mathrm{C}_{23} \mathrm{H}_{34} \mathrm{NaO}_{4}[\mathrm{M}+\mathrm{Na}]+$ calcd. 397.2349, found 397.2357. [a]20D: $+146\left(\mathrm{c} 0.01, \mathrm{CHCl}_{3}\right)$.

\section{$4 \beta$-bromo, $12 \alpha$-acetoxy-5H $\beta$-pregnan-3,20-dione (3)}


To a solution of compound 2 (2.14 g, $5.71 \mathrm{mmol})$ in $\mathrm{AcOH}(60$ $\mathrm{mL})$, was added dropwise over $45 \mathrm{~min}$ a solution of $\mathrm{Br} 2(0.29 \mathrm{~mL}, 5.65$ $\mathrm{mmol})$ in $\mathrm{AcOH}(25 \mathrm{~mL})$. The solution was stirred for an additional $30 \mathrm{~min}$ and concentrated under reduced pressure. The residue was dissolved in $\mathrm{CH}_{2} \mathrm{Cl}_{2}(50 \mathrm{~mL})$ and washed with water $(3 \times 30 \mathrm{~mL})$. The combined aqueous layers were extracted with $\mathrm{CH}_{2} \mathrm{Cl}_{2}(2 \times 50 \mathrm{~mL})$. The combined organic layers were washed with sat. $\mathrm{NaHCO}_{3}(3 \times 30 \mathrm{~mL})$, dried over $\mathrm{Na}_{2} \mathrm{SO}_{4}$, filtered and concentrated under reduced pressure to yield compound 3 as a white solid $\left(2.89 \mathrm{~g}\right.$, quant., mp $\left.167.4-169.9^{\circ} \mathrm{C}\right)$ which was used directly for the next step. IR $\left(\mathrm{ATR}, \mathrm{cm}^{-1}\right): 1726,1698$. ${ }^{1} \mathrm{H}$ NMR $\left(\mathrm{CDCl}_{3}\right)$ \&: $5.16\left(\mathrm{t},{ }^{1} \mathrm{H}, \mathrm{J}=2.4 \mathrm{~Hz}, \mathrm{H}-12 \beta\right) ; 4.92\left(\mathrm{~d},{ }^{1} \mathrm{H}, \mathrm{J}=11.7\right.$ $\mathrm{Hz}, \mathrm{H}-4 \alpha) ; 2.92\left(\mathrm{t},{ }^{1} \mathrm{H}, \mathrm{J}=9.3 \mathrm{~Hz}, \mathrm{H}-17 \alpha\right) ; 2.47$ (dt, $\left.{ }^{1} \mathrm{H}, \mathrm{J}=3.9-14.4 \mathrm{~Hz}\right)$; 2.35-0.60 (17H, m); 2.11 (s, 3H, CH , H-21); 2.00 (s, 3H, CH, Ac); 0.88 $\left(\mathrm{s}, 3 \mathrm{H}, \mathrm{CH}_{3}\right) ; 0.71\left(\mathrm{~s}, 3 \mathrm{H}, \mathrm{CH}_{3}\right) .13 \mathrm{C} \mathrm{NMR}\left(\mathrm{CDCl}_{3}\right) \delta: 208.5(\mathrm{C}=\mathrm{O})$; $201.8(\mathrm{C}=\mathrm{O}) ; 170.2(\mathrm{C}=\mathrm{O}, \mathrm{OAc}) ; 74.1(\mathrm{CH}, \mathrm{C}-12) ; 59.5(\mathrm{CH}) ; 55.4$ $(\mathrm{CH}) ; 53.9(\mathrm{CH}) ; 49.4(\mathrm{CH}) ; 46.6(\mathrm{C}) ; 37.6(\mathrm{C}) ; 36.3\left(\mathrm{CH}_{2}\right) ; 36.2\left(\mathrm{CH}_{2}\right)$; $35.8(\mathrm{CH}) ; 35.4(\mathrm{CH}) ; 31.1(\mathrm{CH}) ; 26.0\left(\mathrm{CH}_{2}\right) ; 25.4\left(\mathrm{CH}_{2}\right) ; 24.7\left(\mathrm{CH}_{2}\right)$; $23.6\left(\mathrm{CH}_{2}\right) ; 2.9\left(\mathrm{CH}_{3}\right) ; 22.4\left(\mathrm{CH}_{2}\right) ; 21.3\left(\mathrm{CH}_{3}\right) ; 13.9\left(\mathrm{CH}_{3}\right)$. HRMS (ESI) $\mathrm{m} / \mathrm{z}: \mathrm{C}_{23} \mathrm{H}_{33} \mathrm{BrNaO}_{4}[\mathrm{M}+\mathrm{Na}]+$ calcd. 477.1437, found 477.1445. [a $]^{20}$ : $+151\left(\right.$ c $\left.0.01, \mathrm{CHCl}_{3}\right)$.

\section{2a-acetoxy-pregn-4-ene-3,20-dione (4)}

To a solution of the crude compound $3(2.60 \mathrm{~g}, 5.73 \mathrm{mmol})$ in DMF $(65 \mathrm{~mL})$, was added $\mathrm{LiCl}(1.20 \mathrm{~g}, 28.24 \mathrm{mmol})$. The reaction mixture was refluxed for $1 \mathrm{~h}$ and the medium was concentrated under reduced pressure. The orange residue was dissolved in $\mathrm{AcOEt}(50 \mathrm{~mL})$ and washed with water $(3 \times 30 \mathrm{~mL})$. The combined aqueous layers were extracted with AcOEt $(3 \times 30 \mathrm{~mL})$. The combined organic layers were washed with brine $(1 \times 30 \mathrm{~mL})$, dried over $\mathrm{Na}_{2} \mathrm{SO}_{4}$, filtered and concentrated under reduced pressure. The crude material was purified by column chromatography (60 g silica gel, petroleum ether-ethyl acetate, 8:2) to afford compound 4 as a slightly yellow solid (1.43 g, 67\%, mp 147.4$\left.151^{\circ} \mathrm{C}\right)$. IR (ATR, $\left.\mathrm{cm}^{-1}\right): 1729,1700,1665,1618 .{ }^{1} \mathrm{H} \mathrm{NMR}\left(\mathrm{CDCl}_{3}\right) \delta$ : $5.71\left(\mathrm{~s},{ }^{1} \mathrm{H}, \mathrm{H}-4\right) ; 5.15$ (t, $\left.{ }^{1} \mathrm{H}, \mathrm{J}=2.7 \mathrm{~Hz}, \mathrm{H}-12 \beta\right) ; 2.91\left(\mathrm{t},{ }^{1} \mathrm{H}, \mathrm{J}=9 \mathrm{~Hz}\right.$, $\mathrm{H}-17 \mathrm{a}) ; 2.40-0.60$ (m, 17H); 2.16 (s, 3H, CH3, $\mathrm{H}-21) ; 2.00$ (s, 3H, $\mathrm{CH}_{3}$, Ac); $1.14\left(\mathrm{~s}, 3 \mathrm{H}, \mathrm{CH}_{3}\right) ; 0.72\left(\mathrm{~s}, 3 \mathrm{H}, \mathrm{CH}_{3}\right) .{ }^{13} \mathrm{C} \mathrm{NMR}\left(\mathrm{CDCl}_{3}\right) \delta: 208.5$ $(\mathrm{C}=\mathrm{O}, \mathrm{C}-20) ; 199.1(\mathrm{C}=\mathrm{O}, \mathrm{C}-3) ; 170.2(\mathrm{C}=\mathrm{C}, \mathrm{C}-5) ; 170.0(\mathrm{C}=\mathrm{O}, \mathrm{OAc})$; 124.1 (CH, C-4); 74.3 (CH, C-12); $55.3(\mathrm{CH}, \mathrm{C}-17) ; 48.7(\mathrm{CH}) ; 47.9$ $(\mathrm{CH}) ; 46.4(\mathrm{C}) ; 38.0(\mathrm{C}) ; 35.7(\mathrm{CH}) ; 35.6(\mathrm{CH} 2) ; 33.8\left(\mathrm{CH}_{2}\right) ; 32.6\left(\mathrm{CH}_{2}\right)$; $31.6\left(\mathrm{CH}_{2}\right) ; 31.1\left(\mathrm{CH}_{3}\right) ; 25.6\left(\mathrm{CH}_{2}\right) ; 23.6\left(\mathrm{CH}_{2}\right) ; 22.2\left(\mathrm{CH}_{2}\right) ; 21.2\left(\mathrm{CH}_{3}\right)$; $17.1\left(\mathrm{CH}_{3}\right) ; 13.7\left(\mathrm{CH}_{3}\right)$. HRMS (ESI) m/z: $\mathrm{C}_{23} \mathrm{H}_{32} \mathrm{NaO}_{4}[\mathrm{M}+\mathrm{Na}]+$ calcd. 395.2193, found 395.2202. [a]20D: +217 (c 0.01, $\mathrm{CHCl}_{3}$ ).

\section{3,12a-diacetoxy-pregna-3,5-diene-20-one (5)}

A solution of compound $4(2.80 \mathrm{~g}, 3.73 \mathrm{mmol})$ in Ac2O $(18 \mathrm{~mL})$ was treated with $\mathrm{AcCl}(30 \mathrm{~mL})$ and heated under reflux for $1 \mathrm{~h}$. After cooling to r.t., the reaction mixture was concentrated under reduced pressure without heating $\left(25^{\circ} \mathrm{C}, 10-2 \mathrm{mbar}\right)$. The orange material was purified by column chromatography ( $100 \mathrm{~g}$ silica gel, petroleum etherethyl acetate, 9:1), to afford compound 5 as a white solid $(2.69 \mathrm{~g}, 85 \%$, mp $134.7-137.5^{\circ} \mathrm{C}$ ). IR (ATR, $\left.\mathrm{cm}^{-1}\right): 1745,1726,1700,1667 .{ }^{1} \mathrm{H}$ NMR $\left(\mathrm{CDCl}_{3}\right) \delta: 5.69\left(\mathrm{~d},{ }^{1} \mathrm{H}, \mathrm{J}=1.8 \mathrm{~Hz}, \mathrm{H}-4\right) ; 5.39\left(\mathrm{~d},{ }^{1} \mathrm{H}, \mathrm{J}=3.6 \mathrm{~Hz}, \mathrm{H}-6\right) ; 5.18$ (t, $\left.{ }^{1} \mathrm{H}, \mathrm{J}=3, \mathrm{H}-12 \beta\right) ; 2.94$ (t, $\left.{ }^{1} \mathrm{H}, \mathrm{J}=8.7, \mathrm{H}-17 \alpha\right) ; 2.50-1.45$ (m, $\left.1^{1} \mathrm{H}\right) ; 2.13$ (s, 3H, CH, $\mathrm{H}-21) ; 2.11$ (s, 3H, $\left.\mathrm{CH}_{3}, \mathrm{Ac}\right) ; 2.03$ (s, 3H, $\left.\mathrm{CH}_{3}, \mathrm{Ac}\right) ; 1.45-$ 0.80 (m, 4H); 0.98 (s, 3H, CH3); 0.74 (3H, s, Me). ${ }^{13} \mathrm{C} \mathrm{NMR}\left(\mathrm{CDCl}_{3}\right) \delta$ : $208.7(\mathrm{C}=\mathrm{O}, \mathrm{C}-20) ; 170.4(\mathrm{C}=\mathrm{O}, \mathrm{OAc}) ; 169.4(\mathrm{C}=\mathrm{O}, \mathrm{OAc}) ; 147.1(\mathrm{C}=\mathrm{C})$; $139.2(\mathrm{C}=\mathrm{C}) ; 132.4(\mathrm{CH}, \mathrm{C}=\mathrm{CH}) ; 116.8(\mathrm{CH}, \mathrm{C}=\mathrm{CH}) ; 74.3(\mathrm{CH}, \mathrm{C}-12)$; $55.4(\mathrm{CH}) ; 49.5(\mathrm{CH}) ; 46.5(\mathrm{C}) ; 42.4(\mathrm{CH}) ; 34.4\left(\mathrm{CH}_{2}\right) ; 33.7(\mathrm{C}) ; 31.5$ $(\mathrm{CH}) ; 31.4\left(\mathrm{CH}_{2}\right) ; 31.1\left(\mathrm{CH}_{3}\right) ; 25.7\left(\mathrm{CH}_{2}\right) ; 24.6\left(\mathrm{CH}_{2}\right) ; 23.6\left(\mathrm{CH}_{2}\right) ; 22.9$ $\left(\mathrm{CH}_{2}\right) ; 21.3\left(\mathrm{CH}_{3}\right) ; 21.1\left(\mathrm{CH}_{3}\right) ; 18.6\left(\mathrm{CH}_{3}\right) ; 13.8\left(\mathrm{CH}_{3}\right)$. HRMS (ESI) $\mathrm{m} / \mathrm{z}: \mathrm{C}_{25} \mathrm{H}_{34} \mathrm{NaO}_{5}[\mathrm{M}+\mathrm{Na}]+$ calcd. 437.2298, found 437.2302. [a]20D: $+2\left(\mathrm{c} 0.01, \mathrm{CHCl}_{3}\right)$.

\section{3,12a-diacetoxy, cyclic 20-(ethylene acetal)-pregna-3,5-diene} (5a)

To a solution of compound $5(635 \mathrm{mg}, 1.53 \mathrm{mmol})$ in toluene ( 25 $\mathrm{mL})$, were added ethylene glycol $(1.10 \mathrm{~mL}, 20.03 \mathrm{mmol})$ and PPTS (catalytic amount). The reaction mixture was refluxed (Dean Stark trap) for $3.5 \mathrm{~h}$. The solvent was evaporated under reduced pressure and the residue dissolved in $\mathrm{CH} 2 \mathrm{Cl} 2(20 \mathrm{~mL})$ was washed with water $(2 \times 20$ $\mathrm{mL})$. The combined aqueous layers were extracted with $\mathrm{CH} 2 \mathrm{Cl} 2(2 \times 20$ $\mathrm{mL})$. The combined organic layers were washed with sat. $\mathrm{NaHCO}_{3}(1 \times$ $20 \mathrm{~mL}$ ), dried over $\mathrm{Na} 2 \mathrm{SO}$, filtered and concentrated under reduced pressure. The crude material was purified by column chromatography (30 g silica gel, petroleum ether-ethyl acetate, 9:1) to afford compound $5 \mathrm{a}$ as a yellow solid $\left(700 \mathrm{mg}\right.$, quant., $\left.\mathrm{mp} 68-69^{\circ} \mathrm{C}\right)$. IR (ATR, $\left.\mathrm{cm}^{-1}\right)$ : 1733-1680. ${ }^{1} \mathrm{H}$ NMR $\left(\mathrm{CDCl}_{3}\right) \delta: 5.69\left(\mathrm{~s},{ }^{1} \mathrm{H}, \mathrm{H}-6\right) ; 5.40\left(\mathrm{~d},{ }^{1} \mathrm{H}, \mathrm{J}=3.0 \mathrm{~Hz}\right.$, $\mathrm{H}-4) ; 5.14$ (t, $\left.{ }^{1} \mathrm{H}, \mathrm{J}=2.4 \mathrm{~Hz}, \mathrm{H}-12 \beta\right) ; 4.00-3.80$ (m, 4H, $\left.\mathrm{OCH}_{2}\right) ; 2.50-1.90$ $(\mathrm{m}, 4 \mathrm{H}) ; 2.13\left(\mathrm{~s}, 3 \mathrm{H}, \mathrm{CH}_{3}, \mathrm{Ac}\right) ; 2.04\left(\mathrm{~s}, 3 \mathrm{H}, \mathrm{CH}_{3}, \mathrm{Ac}\right) ; 1.90-1.40(\mathrm{~m}$, $10 \mathrm{H}) ; 1.40-0.90(\mathrm{~m}, 2 \mathrm{H}) ; 1.22$ (s, 3H, $\left.\mathrm{CH}_{3}, \mathrm{H}-21\right) ; 0.99$ (s, 3H, $\mathrm{CH}_{3}$ ); $0.88\left(\mathrm{~s}, 3 \mathrm{H}, \mathrm{CH}_{3}\right) .{ }^{13} \mathrm{C} \mathrm{NMR}\left(\mathrm{CDCl}_{3}\right) \delta: 170.6(\mathrm{C}=\mathrm{O}, \mathrm{OAc}) ; 169.4(\mathrm{C}=\mathrm{O}$, OAc); $147.0(\mathrm{C}=\mathrm{C}) ; 139.3(\mathrm{C}=\mathrm{C}) ; 123.7(\mathrm{C}=\mathrm{CH}) ; 116.7(\mathrm{C}=\mathrm{CH}) ; 111.4$ (C, C-2); $75.3(\mathrm{CH}, \mathrm{C}-12) ; 64.9\left(\mathrm{OCH}_{2}\right) ; 63.3\left(\mathrm{OCH}_{2}\right) ; 49.7(\mathrm{CH}) ; 49.2$ $(\mathrm{CH}) ; 44.3(\mathrm{C}, 13-\mathrm{C}) ; 42.5(\mathrm{CH}) ; 34.4\left(\mathrm{CH}_{2}\right) ; 33.7(10-\mathrm{C}) ; 31.4\left(\mathrm{CH}_{2}\right)$ $31.0(\mathrm{CH}) ; 25.4\left(\mathrm{CH}_{2}\right) ; 24.7\left(\mathrm{CH}_{2}\right) ; 24.0(\mathrm{CH}) ; 23.1\left(\mathrm{CH}_{2}\right) ; 22.3\left(\mathrm{CH}_{2}\right)$; $21.4\left(\mathrm{CH}_{3}\right) ; 21.1\left(\mathrm{CH}_{3}\right) ; 18.6\left(\mathrm{CH}_{3}\right) ; 13.5\left(\mathrm{CH}_{3}\right)$. HRMS (ESI) m/z: $\mathrm{C}_{27} \mathrm{H}_{38} \mathrm{NaO}_{6}[\mathrm{M}+\mathrm{Na}]+$ calcd. 481.2561, found 481.2569. [a]20D: +61 (c $0.02, \mathrm{MeOH})$.

\section{$3 \beta$ hydroxy, 12 $\alpha$-acetoxy, cyclic 20 -(ethylene acetal) pregn-5- ene $(5 b)$}

To a suspension of $\mathrm{NaBH} 4(860 \mathrm{mg}, 22.7 \mathrm{mmol})$ in a mixture of $\mathrm{MeOH}(6 \mathrm{~mL})$ and THF $(6 \mathrm{~mL})$, was added a solution of compound $5 \mathrm{a}$ (540 mg, $1.18 \mathrm{mmol})$ in THF $(5 \mathrm{~mL})$. The reaction mixture was stirred for $1.5 \mathrm{~h}$ at r.t. and treated with $10 \% \mathrm{HCl}$ until neutral $\mathrm{pH}$. $\mathrm{CH}_{2} \mathrm{Cl}_{2}(15$ $\mathrm{mL}$ ) was added and the layers were separated. The aqueous layer was extracted with $\mathrm{CH}_{2} \mathrm{Cl}_{2}(3 \times 15 \mathrm{~mL})$. The combined organic phase were dried over $\mathrm{Na}_{2} \mathrm{SO}_{4}$, filtered and concentrated under reduced pressure. The crude material was purified by column chromatography ( $30 \mathrm{~g}$ silica gel, petroleum ether-ethyl acetate, 7:3), to afford compound $5 \mathrm{~b}$ as a white solid $\left(333 \mathrm{mg}, 70 \%, \mathrm{mp} 166-167^{\circ} \mathrm{C}\right)$. IR (ATR, $\left.\mathrm{cm}^{-1}\right)$ : 3446, 1699; ${ }^{1} \mathrm{H} \mathrm{NMR}\left(\mathrm{CDCl}_{3}\right) \delta: 5.55\left(\mathrm{~s},{ }^{1} \mathrm{H}, \mathrm{H}-6\right) ; 5.11\left(\mathrm{t},{ }^{1} \mathrm{H}, \mathrm{J}=2.7 \mathrm{~Hz}, \mathrm{H}-12 \beta\right) ; 4.24$ (t, $\left.{ }^{1} \mathrm{H}, \mathrm{J}=2.4 \mathrm{~Hz}, \mathrm{H}-3 \mathrm{a}\right) ; 4.00-3.70\left(\mathrm{~m}, 4 \mathrm{H}, \mathrm{OCH}_{2}\right) ; 2.28$ (t, ${ }^{1} \mathrm{H}, \mathrm{J}=9.9$ $\mathrm{Hz}, \mathrm{H}-17 \mathrm{\alpha}) ; 2.10-1.90$ (m, 3H); 2.04 (s, 3H, $\left.\mathrm{CH}_{3}, \mathrm{Ac}\right) ; 1.90-0.70$ (m, $15 \mathrm{H}) ; 1.23$ (s, 3H, $\left.\mathrm{CH}_{3}, \mathrm{H}-21\right) ; 1.20\left(\mathrm{~s}, 3 \mathrm{H}, \mathrm{CH}_{3}\right) ; 0.88\left(\mathrm{~s}, 3 \mathrm{H}, \mathrm{CH}_{3}\right) .{ }^{13} \mathrm{C}$ $\operatorname{NMR}\left(\mathrm{CDCl}_{3}\right) \delta: 170.5(\mathrm{C}=\mathrm{O}, \mathrm{OAc}) ; 147.1(\mathrm{C}=\mathrm{C}, \mathrm{C}-5) ; 129.0(\mathrm{C}=\mathrm{CH}$, C-6); 111.4 (C, C-20); 75.2 (CH, C-3); $74.0(\mathrm{CH}, \mathrm{C}-12) ; 67.8(\mathrm{CH}) ; 64.9$ $\left(\mathrm{OCH}_{2}\right) ; 63.3\left(\mathrm{OCH}_{2}\right) ; 49.7(\mathrm{CH}) ; 48.5(\mathrm{CH}) ; 48.4(\mathrm{C}) ; 44.4\left(\mathrm{CH}_{2}\right) ; 38.6$ $\left(\mathrm{CH}_{2}\right) ; 36.8(\mathrm{C}) ; 36.2\left(\mathrm{CH}_{2}\right) ; 29.6(\mathrm{CH}) ; 29.1\left(\mathrm{CH}_{2}\right) ; 25.2\left(\mathrm{CH}_{2}\right) ; 24.0$ $\left(\mathrm{CH}_{3}\right) ; 23.0\left(\mathrm{CH}_{2}\right) ; 22.3\left(\mathrm{CH}_{2}\right) ; 21.4\left(\mathrm{CH}_{3}\right) ; 21.2\left(\mathrm{CH}_{3}\right) ; 13.5(\mathrm{CH} 3)$ HRMS (ESI) $\mathrm{m} / \mathrm{z}: \mathrm{C}_{25} \mathrm{H}_{39} \mathrm{O}_{5}[\mathrm{M}+\mathrm{H}]+$ calcd. 419.2680, found 419.2745 . [a]20D:+58 (c 0.01, $\left.\mathrm{CHCl}_{3}\right)$.

\section{$3 \beta, 12 \alpha$-dihydroxy, cyclic 20-(ethylene acetal) pregn-5-ene (6)}

A solution of compound $5 \mathrm{~b}$ (330 mg, $1.15 \mathrm{mmol}$ ) in $\mathrm{MeOH}(15$ $\mathrm{mL})$ was treated with $\mathrm{KOH}(83 \mathrm{mg}, 1.48 \mathrm{mmol})$ and refluxed for $1 \mathrm{~h}$. After cooling to r.t., water $(20 \mathrm{~mL})$ was added and the aqueous layer was extracted with $\mathrm{CH}_{2} \mathrm{Cl}_{2}(3 \times 20 \mathrm{~mL})$. The combined organic layers were dried over $\mathrm{Na}_{2} \mathrm{SO}_{4}$, filtered and concentrated under reduced pressure to afford compound 6 as a white solid $\left(266 \mathrm{mg}, 85 \%\right.$, mp $\left.133.0-135.2^{\circ} \mathrm{C}\right)$ 
Citation: Geoffroy P, Ressault B, Miesch M (2015) Synthesis of Hoodigogenin A, the Aglycone of Hoodigosides Extracted from Hoodia gordonii. J Steroids Horm Sci 6: 163. doi:10.4172/2157-7536.1000163

Page 4 of 5

which was used directly for the next step. IR $\left(\right.$ ATR $\left.\mathrm{cm}^{-1}\right): 3460,1733 .{ }^{1} \mathrm{H}$ $\operatorname{NMR}\left(\mathrm{C}_{6} \mathrm{D}_{6}\right) \delta: 5.34\left(\mathrm{~m},{ }^{1} \mathrm{H}, \mathrm{H}-6\right) ; 4.01\left(\mathrm{t},{ }^{1} \mathrm{H}, \mathrm{J}=3 \mathrm{~Hz}, \mathrm{H}-12 \beta\right) ; 3.55-3.25$ $\left(\mathrm{m}, 5 \mathrm{H}, \mathrm{OCH}_{2}+\mathrm{H}-3 \alpha\right) ; 2.37\left(\mathrm{t},{ }^{1} \mathrm{H}, \mathrm{J}=9.96 \mathrm{~Hz}, \mathrm{H}-17 \alpha\right) ; 2.30-2.20(\mathrm{~m}$, $3 \mathrm{H}) ; 2.05-0.80(\mathrm{~m}, 16 \mathrm{H}) ; 1.021\left(\mathrm{~s}, 3 \mathrm{H}, \mathrm{CH}_{3}, \mathrm{H}-21\right) ; 0.92\left(\mathrm{~s}, 3 \mathrm{H}, \mathrm{CH}_{3}\right) ;$ $0.87\left(\mathrm{~s}, 3 \mathrm{H}, \mathrm{CH}_{3}\right) \cdot{ }^{13} \mathrm{C}$ NMR $\left(\mathrm{C}_{6} \mathrm{D}_{6}\right) \delta: 140.6(\mathrm{C}=\mathrm{C}, \mathrm{C}-5) ; 121.6(\mathrm{C}=\mathrm{CH}$, $\mathrm{C}-6) ; 112.2(\mathrm{C}, \mathrm{C}-20) ; 72.7(\mathrm{CH}, \mathrm{C}-12) ; 71.7(\mathrm{CH}, \mathrm{C}-3) ; 64.0(\mathrm{OCH} 2)$ $63.7\left(\mathrm{OCH}_{2}\right) ; 49.7(\mathrm{CH}) ; 48.0(\mathrm{CH}) ; 45.8(\mathrm{C}) ; 44.2(\mathrm{CH}) ; 42.3\left(\mathrm{CH}_{2}\right)$; $37.0\left(\mathrm{CH}_{2}\right) ; 36.1(\mathrm{C}) ; 31.6(\mathrm{CH}) ; 31.3\left(\mathrm{CH}_{2}\right) ; 31.2\left(\mathrm{CH}_{2}\right) ; 27.6\left(\mathrm{CH}_{2}\right)$; $23.8\left(\mathrm{CH}_{3}\right) ; 23.1\left(\mathrm{CH}_{2}\right) ; 22.9\left(\mathrm{CH}_{2}\right) ; 19.2\left(\mathrm{CH}_{3}\right) ; 13.9\left(\mathrm{CH}_{3}\right)$. HRMS (ESI) $\mathrm{m} / \mathrm{z}: \mathrm{C}_{23} \mathrm{H}_{36} \mathrm{NaO}_{4}[\mathrm{M}+\mathrm{Na}]+$ calcd. 399.2506, found 399.2518, [a]20D: -25 (c $0.0075, \mathrm{CHCl}_{3}$ ).

\section{$3 \beta$-acetoxy,12a-hydroxy, cyclic 20-(ethylene acetal) pregn-5-} ene (7)

To a solution of compound $6(564 \mathrm{mg}, 1.51 \mathrm{mmol})$ in $\mathrm{CH}_{2} \mathrm{Cl}_{2}(25$ $\mathrm{mL})$, were added $\mathrm{Ac}_{2} \mathrm{O}(0.17 \mathrm{~mL}, 1.81 \mathrm{mmol})$, pyridine $(0.24 \mathrm{~mL}, 3.02$ mmol) and DMAP (catalytic amount). The reaction mixture was stirred at r.t. for $3 \mathrm{~h}$ and treated with water $(20 \mathrm{~mL})$. The aqueous layer was extracted with $\mathrm{CH}_{2} \mathrm{Cl}_{2}(3 \times 20 \mathrm{~mL})$. The combined organic layers were washed with $10 \% \mathrm{HCl}(1 \times 20 \mathrm{~mL})$, with sat. $\mathrm{NaHCO}_{3}(1 \times 20 \mathrm{~mL})$, dried over $\mathrm{Na}_{2} \mathrm{SO}_{4}$, filtered and concentrated under reduced pressure. The crude material was purified by column chromatography ( $30 \mathrm{~g}$ silica gel, petroleum ether-ethyl acetate, 9:1) to afford compound 7 as a white solid (505 mg, 80\%, mp 158.8-160.6 ${ }^{\circ} \mathrm{C}$ ). IR (ATR, $\left.\mathrm{cm}^{-1}\right): 3417,1733$. ${ }^{1} \mathrm{H}$ NMR $\left(\mathrm{C}_{6} \mathrm{D}_{6}\right) \delta: 5.40-5.33\left(\mathrm{~m},{ }^{1} \mathrm{H}, \mathrm{H}-6\right) ; 4.81\left(\mathrm{tt},{ }^{1} \mathrm{H}, \mathrm{J}=4.8-11.3 \mathrm{~Hz}\right.$, $\mathrm{H}-3 \alpha) ; 4.01$ (t, $\left.{ }^{1} \mathrm{H}, \mathrm{J}=2.4 \mathrm{~Hz}, \mathrm{H}-12 \beta\right) ; 3.48$ (s, 4H, OCH2); 2.60-2.30 $(\mathrm{m}, 3 \mathrm{H}) ; 2.00-0.80(\mathrm{~m}, 16 \mathrm{H}) ; 1.75$ (s, 3H, CH3, Ac); 1.22 (s, 3H, $\mathrm{CH}_{3}$, $\mathrm{H}-21) ; 0.89$ (s, 3H, $\left.\mathrm{CH}_{3}\right) ; 0.86\left(\mathrm{~s}, 3 \mathrm{H}, \mathrm{CH}_{3}\right) .{ }^{13} \mathrm{C} \mathrm{NMR}\left(\mathrm{C}_{6} \mathrm{D}_{6}\right) \delta: 169.3$ $(\mathrm{C}=\mathrm{O}, \mathrm{OAc}) ; 139.5$ (C=C, C-5); 122.5 (C=CH, C-6); 111.9 (C, C-20); $73.6(\mathrm{CH}, \mathrm{C}-12) ; 72.2(\mathrm{CH}, \mathrm{C}-3) ; 64.0\left(\mathrm{OCH}_{2}\right) ; 63.2\left(\mathrm{OCH}_{2}\right) ; 49.6$ $(\mathrm{CH}) ; 47.8(\mathrm{CH}) ; 45.8(\mathrm{C}, 13-\mathrm{C}) ; 43.9(\mathrm{CH}) ; 38.3\left(\mathrm{CH}_{2}\right) ; 36.7\left(\mathrm{CH}_{2}\right)$; 36.1 (C, 10-C); $31.5(\mathrm{CH} 2) ; 31.2(\mathrm{CH}) ; 27.8\left(\mathrm{CH}_{2}\right) ; 23.6\left(\mathrm{CH}_{3}\right) ; 23.1$ $\left(\mathrm{CH}_{2}\right) ; 22.7\left(\mathrm{CH}_{2}\right) ; 20.7\left(\mathrm{CH}_{3}\right) ; 18.8\left(\mathrm{CH}_{3}\right) ; 13.8\left(\mathrm{CH}_{3}\right)$. HRMS (ESI) $\mathrm{m} / \mathrm{z}: \mathrm{C}_{25} \mathrm{H}_{38} \mathrm{NaO}_{5}[\mathrm{M}+\mathrm{Na}]+$ calcd. 441.2611, found 441.2608, [a]20D: $-31\left(\mathrm{c} 0.006, \mathrm{CHCl}_{3}\right)$.

\section{$3 \beta$-acetoxy,12-keto, cyclic 20-(ethylene acetal) pregn-5-ene} (8)

To a solution of compound 7 (143 $\mathrm{mg}, 0.34 \mathrm{mmol})$ in $\mathrm{CH}_{2} \mathrm{Cl}_{2}$ (3 $\mathrm{mL})$ at $0^{\circ} \mathrm{C}$, was added the Dess Martin reagent $(174 \mathrm{mg}, 0.41 \mathrm{mmol})$. The reaction mixture was stirred for $1 \mathrm{~h}$ at r.t., then was treated with sat. $\mathrm{Na}_{2} \mathrm{~S}_{2} \mathrm{O}_{3}(8 \mathrm{~mL})$ and the reaction mixture was stirred for $5 \mathrm{~min}$. To the medium was added sat. $\mathrm{NaHCO}_{3}(8 \mathrm{~mL})$ and the reaction mixture was stirred for $5 \mathrm{~min}$. The layers were separated and the aqueous layer was extracted with $\mathrm{CH}_{2} \mathrm{Cl}_{2}(3 \times 10 \mathrm{~mL})$. The combined organic layers were dried over $\mathrm{Na}_{2} \mathrm{SO}_{4}$, filtered and concentrated under reduced pressure. The crude material was purified by column chromatography ( $5 \mathrm{~g}$ silica gel, cyclohexane-ethyl acetate, 7:3) to afford compound 8 as a white solid (140 mg, $99 \%$, mp 150-151 ${ }^{\circ} \mathrm{C}$ ). IR (ATR, $\left.\mathrm{cm}^{-1}\right): 1722,1700 .{ }^{1} \mathrm{H}$ NMR $\left(\mathrm{CDCl}_{3}\right) \delta: 5.40\left(\mathrm{~m},{ }^{1} \mathrm{H}, \mathrm{H}-6\right) ; 4.58\left(\mathrm{tt},{ }^{1} \mathrm{H}, \mathrm{J}=4.6-1.4 \mathrm{~Hz}, \mathrm{H}-3 \alpha\right)$; 4.05-3.65 (m, 4H, $\left.\mathrm{OCH}_{2}\right) ; 2.80-2.55(\mathrm{~m}, 2 \mathrm{H}) ; 2.45-1.95(\mathrm{~m}, 4 \mathrm{H}) ; 2.3$ (s, 3H, $\left.\mathrm{CH}_{3}, \mathrm{Ac}\right) ; 1.95-1.10(\mathrm{~m}, 12 \mathrm{H}) ; 1.25$ (s, 3H, CH, $\left.\mathrm{H}-21\right) ; 1.15$ (s, $\left.3 \mathrm{H}, \mathrm{CH}_{3}\right) ; 1.12\left(\mathrm{~s}, 3 \mathrm{H}, \mathrm{CH}_{3}\right) .{ }^{13} \mathrm{C}$ NMR $\left(\mathrm{CDCl}_{3}\right) \delta: 214.0(\mathrm{C}=\mathrm{O}, \mathrm{C}-12)$; $170.4(\mathrm{C}=\mathrm{O}, \mathrm{OAc}) ; 139.4(\mathrm{C}=\mathrm{C}, \mathrm{C}-5) ; 122.3(\mathrm{C}=\mathrm{CH}, \mathrm{C}-6) ; 111.2(\mathrm{C}$, C-20); $73.5(\mathrm{CH}, \mathrm{C}-3) ; 65.5(\mathrm{OCH} 2) ; 62.9\left(\mathrm{OCH}_{2}\right) ; 58.3(\mathrm{CH}) ; 56.6(\mathrm{C}$, C-13); $53.6(\mathrm{CH}) ; 49.1(\mathrm{CH}) ; 37.9(\mathrm{CH} 2) ; 37.7\left(\mathrm{CH}_{2}\right) ; 37.5(\mathrm{C}, \mathrm{C}-10)$; $36.7\left(\mathrm{CH}_{2}\right) ; 31.4(\mathrm{CH}) ; 31.3\left(\mathrm{CH}_{2}\right) ; 27.5\left(\mathrm{CH}_{2}\right) ; 4.4\left(\mathrm{CH}_{3}\right) ; 24.0\left(\mathrm{CH}_{2}\right)$; $22.0\left(\mathrm{CH}_{2}\right) ; 21.4\left(\mathrm{CH}_{3}\right) ; 18.9\left(\mathrm{CH}_{3}\right) ; 12.9\left(\mathrm{CH}_{3}\right)$. HRMS (ESI) m/z: $\mathrm{C}_{25} \mathrm{H}_{36} \mathrm{NaO}_{5}[\mathrm{M}+\mathrm{Na}]+$ calcd. 439.2455, found 439.2496, [a]20D: +12 (c $0.01, \mathrm{CHCl}_{3}$ ).

\section{Synthesis of compounds 10, 11 and 12}

A solution of compound 8 ( $895 \mathrm{mg}, 2.15 \mathrm{mmol})$ in $\mathrm{CH}_{2} \mathrm{Cl}_{2}(280$ $\mathrm{mL}$ ) was degassed with argon for $15 \mathrm{~min}$ and then was irradiated with a high pressure mercury lamp Philips HPK 125 for 15 min in a quartz vessel. The solvent was then evaporated under reduced pressure, to afford the crude seco aldehyde 9 (931 mg, 100\%).

To a solution of crude seco aldehyde $(931 \mathrm{mg}$, max. $2.15 \mathrm{mmol})$ in THF $(4.0 \mathrm{~mL})$, was added a solution of $\mathrm{AcOH} / \mathrm{H}_{2} \mathrm{O} / \mathrm{CF}_{3} \mathrm{COOH}$ $(2 / 1 / 0.6,20.0 \mathrm{~mL})$. The reaction mixture was stirred $1.5 \mathrm{~h}$ at $\mathrm{r}$. t.. The reaction mixture was then diluted with $\mathrm{CH}_{2} \mathrm{Cl}_{2}(30 \mathrm{~mL})$ and washed with water $(2 \times 30 \mathrm{~mL})$, with sat. $\mathrm{NaHCO}_{3}(3 \times 30 \mathrm{~mL})$, dried over $\mathrm{Na}_{2} \mathrm{SO}_{4}$, filtered and concentrated under reduced pressure. The crude material was purified by flash column chromatography ( $30 \mathrm{~g}$, silica gel, petroleum ether-ethyl acetate, 8:2 to 6:4) affording compound 10 (153 $\mathrm{mg}, 19 \%$, mp $\left.192-194^{\circ} \mathrm{C}\right)$, compound $11\left(118 \mathrm{mg}, 15 \%\right.$, mp $\left.116-117^{\circ} \mathrm{C}\right)$ and compound $12\left(210 \mathrm{mg}, 25 \%, \mathrm{mp} 140-142^{\circ} \mathrm{C}\right)$.

\section{$3 \beta$-acetoxy,pregn-5-ene-12,20-dione (10)}

IR $\left(\mathrm{ATR}, \mathrm{cm}^{-1}\right): 1729,1703 .{ }^{1} \mathrm{H}$ NMR $\left(\mathrm{CDCl}_{3}\right): 5.42-5.40\left(\mathrm{~m},{ }^{1} \mathrm{H}\right.$ $\mathrm{H}-6) ; 4.59$ (tt, $\left.{ }^{1} \mathrm{H}, \mathrm{J}=4.6-11.4 \mathrm{~Hz}, \mathrm{H}-3 \alpha\right) ; 3.34\left(\mathrm{t},{ }^{1} \mathrm{H}, \mathrm{J}=9.6 \mathrm{~Hz}, \mathrm{H}-17 \alpha\right)$ $2.62\left(\mathrm{t},{ }^{1} \mathrm{H}, \mathrm{J}=13.2 \mathrm{~Hz}, \mathrm{H}-11\right) ; 2.50-2.00(\mathrm{~m}, 4 \mathrm{H}) ; 2.26\left(\mathrm{~s}, 3 \mathrm{H}, \mathrm{CH}_{3}\right.$ $\mathrm{H}-21) ; 2.03$ (s, 3H, CH, $\mathrm{Ac}) ; 2.00-0.80(\mathrm{~m}, 12 \mathrm{H}) ; 1.12\left(\mathrm{~s}, 3 \mathrm{H}, \mathrm{CH}_{3}\right)$; 0,98 (s, 3H, $\left.\mathrm{CH}_{3}\right) \cdot{ }^{13} \mathrm{C} \mathrm{NMR}\left(\mathrm{CDCl}_{3}\right): 213.0(\mathrm{C}=\mathrm{O}) ; 209.4(\mathrm{C}=\mathrm{O}) ; 170.2$ $(\mathrm{C}=\mathrm{O}, \mathrm{OAc}) ; 139.2(\mathrm{C}=\mathrm{C}, \mathrm{C}-5) ; 121.9(\mathrm{C}=\mathrm{CH}, \mathrm{C}-6) ; 73.1(\mathrm{CH}, \mathrm{C}-3)$; $57.6(\mathrm{C}, \mathrm{C}-13) ; 57.4(\mathrm{CH}) ; 54.0(\mathrm{CH}) ; 527(\mathrm{CH}) ; 52.7(\mathrm{CH}) ; 37.6\left(\mathrm{CH}_{2}\right)$; $37.4\left(\mathrm{CH}_{2}\right) ; 37.2(\mathrm{C}, \mathrm{C}-10) ; 36.4\left(\mathrm{CH}_{2}\right) ; 31.1\left(2 \mathrm{CH}+\mathrm{CH}_{2}\right) ; 27.3\left(\mathrm{CH}_{2}\right)$ $24.0\left(\mathrm{CH}_{2}\right) ; 22.4\left(\mathrm{CH}_{2}\right) ; 21.2\left(\mathrm{CH}_{3}\right) ; 18.7\left(\mathrm{CH}_{3}\right) ; 13.2\left(\mathrm{CH}_{3}\right)$. [a]20D: +55 (c $0.009, \mathrm{CHCl}_{3}$ ).

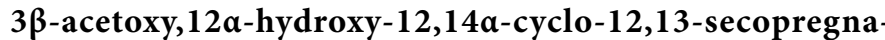 5,13(17)-diene-20-one (11)}

IR (ATR, $\left.\mathrm{cm}^{-1}\right): 3430,1729,1666,1608 .{ }^{1} \mathrm{H}$ NMR $\left(\mathrm{CDCl}_{3}\right): 5.36$ (d, $\left.{ }^{1} \mathrm{H}, \mathrm{J}=4.8 \mathrm{~Hz}, \mathrm{H}-6\right) ; 4.58$ (tt, $\left.{ }^{1} \mathrm{H}, \mathrm{J}=4.8-11.4 \mathrm{~Hz}, \mathrm{H}-3 \alpha\right) ; 4.14\left(\mathrm{dt},{ }^{1} \mathrm{H}\right.$ $\mathrm{J}=8.2-9.7 \mathrm{~Hz}, \mathrm{H}-12 \beta) ; 2.80-0.80(\mathrm{~m}, 17 \mathrm{H}) ; 2.22\left(\mathrm{~s}, 3 \mathrm{H}, \mathrm{CH}_{3}, \mathrm{H}-21\right) ; 2.02$ (s, 3H, CH, Ac); 1.96 (t, 3H, J=2.1 Hz, CH, H-18); 1.04 (s, 3H, CH H-19). ${ }^{13} \mathrm{C}$ NMR $\left(\mathrm{CDCl}_{3}\right): 198.7$ (C=O, C-20); 170.5 (C=O, OAc); 153.7 $(\mathrm{C}=\mathrm{C}) ; 140.5(\mathrm{C}=\mathrm{C}) ; 137.9(\mathrm{C}=\mathrm{C}) ; 122.6(\mathrm{C}=\mathrm{CH}) ; 76.5(\mathrm{CH}, \mathrm{C}-12)$; 73.7 (CH, C-3); 65.7 (C, C-14); $48.3(\mathrm{CH}) ; 39.9(\mathrm{CH}) ; 37.9\left(\mathrm{CH}_{2}\right) ; 37.7$ $\left(\mathrm{CH}_{2}\right) ; 37.2(\mathrm{C}, \mathrm{C}-10) ; 32.4\left(\mathrm{CH}_{2}\right) ; 30.5(\mathrm{CH}) ; 30.3\left(\mathrm{CH}_{2}\right) ; 27.4\left(\mathrm{CH}_{2}\right)$; $25.9\left(\mathrm{CH}_{2}\right) ; 21.8\left(\mathrm{CH}_{2}\right) ; 21.4\left(\mathrm{CH}_{3}\right) ; 18.0\left(\mathrm{CH}_{3}\right) ; 12.1\left(\mathrm{CH}_{3}\right)$. [a] $20 \mathrm{D}:-12$ (c $0.006, \mathrm{CHCl}_{3}$ ).

\section{$3 \beta$-acetoxy, $12 \beta, 14 \beta$-dihydroxy pregn-5-ene-20-one (12)}

IR (ATR, $\left.\mathrm{cm}^{-1}\right): 3399,1730,1686 ;{ }^{1} \mathrm{H}$ NMR $\left(\mathrm{CDCl}_{3}\right) \delta: 5.42(\mathrm{~m}$ $\left.{ }^{1} \mathrm{H}, \mathrm{H}-6\right)$; 4.65-4.50 (m, $\left.{ }^{1} \mathrm{H}, \mathrm{H}-3 \alpha\right) ; 4.37\left(\mathrm{~s},{ }^{1} \mathrm{H} \mathrm{OH}\right) ; 3.61\left(\mathrm{t},{ }^{1} \mathrm{H}, \mathrm{J}=6.3\right.$ $\mathrm{Hz}, \mathrm{H}-17 \mathrm{\alpha}$ ); 3.35 (dd, $\left.{ }^{1} \mathrm{H}, \mathrm{J}=4.2-11.7 \mathrm{~Hz}, \mathrm{H}-12 \alpha\right) ; 2.40-2.15$ (m, 3H); 2.27 (s, 3H, CH, $\mathrm{H}-21) ; 2.10-0.80(\mathrm{~m}, 16 \mathrm{H}) ; 2.03$ (s, 3H, $\left.\mathrm{CH}_{3}, \mathrm{Ac}\right) ; 1.01$ (s, 3H, $\left.\mathrm{CH}_{3}, \mathrm{H}-18\right) ; 0.92$ (s, $\left.3 \mathrm{H}, \mathrm{CH}_{3}, \mathrm{H}-19\right) .{ }^{13} \mathrm{C} \mathrm{NMR}\left(\mathrm{CDCl}_{3}\right) \delta:{ }^{13} \mathrm{C}$ NMR $\left(\mathrm{CDCl}_{3}\right): 218.0(\mathrm{C}=\mathrm{O}, \mathrm{C}-20) ; 170.6(\mathrm{C}=\mathrm{O}, \mathrm{OAc}) ; 137.8(\mathrm{C}=\mathrm{C}$, C-5); $123.1(\mathrm{C}=\mathrm{CH}, \mathrm{C}-6) ; 85.5(\mathrm{C}, \mathrm{C}-14) ; 73.6(\mathrm{CH}, \mathrm{C}-12) ; 73.4(\mathrm{CH}$ C-3); 56.9 (CH, C-17); 55.0 (C, C-13); $43.3(\mathrm{CH}, \mathrm{C}-9) ; 37.8\left(\mathrm{CH}_{2}, \mathrm{C}-1\right)$; $37.0\left(\mathrm{CH}_{2}, \mathrm{C}-4\right) ; 36.8(\mathrm{C}, \mathrm{C}-10) ; 35.6\left(\mathrm{CH}_{3}, \mathrm{C}-21\right) ; 34.5\left(\mathrm{CH}_{2}, \mathrm{C}-15\right)$; $33.1(\mathrm{CH}, \mathrm{C}-8) ; 29.9\left(\mathrm{CH}_{2}, \mathrm{C}-11\right) ; 27.6\left(\mathrm{CH}_{2}, \mathrm{C}-2\right) ; 27 .\left(\mathrm{CH}_{2}, \mathrm{C}-7\right) ; 24.4$ $\left(\mathrm{CH}_{2}, \mathrm{C}-16\right) ; 21.4\left(\mathrm{CH}_{3}, \mathrm{Ac}\right) ; 19.3\left(\mathrm{CH}_{3}, \mathrm{C}-19\right) ; 8.3\left(\mathrm{CH}_{3}, \mathrm{C}-18\right)$. HRMS (ESI) $\mathrm{m} / \mathrm{z}: \mathrm{C}_{23} \mathrm{H}_{34} \mathrm{NaO}_{5}[\mathrm{M}+\mathrm{Na}]+$ calcd. 413.2298, found 413.2308, [a]20D: + 14 (c 0.01, $\mathrm{CHCl}_{3}$ ).

12-O- $\beta$-tigloyl-3 $\beta$-acetoxy, 14 $\beta$-hydroxy-pregn-5-ene-20-one (13) 
Citation: Geoffroy P, Ressault B, Miesch M (2015) Synthesis of Hoodigogenin A, the Aglycone of Hoodigosides Extracted from Hoodia gordonii. J Steroids Horm Sci 6: 163. doi:10.4172/2157-7536.1000163

Page 5 of 5

To a solution of compound $12(40 \mathrm{mg}, 0.10 \mathrm{mmol})$ in $\mathrm{CH}_{2} \mathrm{Cl}_{2}(5$ $\mathrm{mL})$, were added tigloyl chloride $(0.12 \mathrm{~mL}, 1.09 \mathrm{mmol})$, pyridine $(0.14$ $\mathrm{mL}, 1.74 \mathrm{mmol}$ ) and DMAP (catalytic amount). The reaction mixture was heated under reflux for $7 \mathrm{~h}$ and stirred for $15 \mathrm{~h}$ at r.t. Water $(10$ $\mathrm{mL}$ ) was added and the layers were separated. The aqueous layer was extracted with $\mathrm{CH}_{2} \mathrm{Cl}_{2}(2 \times 10 \mathrm{~mL})$. The combined organic layers were washed with $10 \% \mathrm{HCl}(1 \times 10 \mathrm{~mL})$, with sat. $\mathrm{NaHCO}_{3}(1 \times 10 \mathrm{~mL})$, dried over $\mathrm{Na}_{2} \mathrm{SO}_{4}$, filtered and concentrated under reduced pressure. The crude material was purified by column chromatography ( $5 \mathrm{~g}$ silica gel, cyclohexane-ethyl acetate, (95:5) to afford compound 13 as a white solid $\left(38 \mathrm{mg}, 80 \%, \mathrm{mp} 70-72^{\circ} \mathrm{C}\right)$. IR $\left(\mathrm{ATR}, \mathrm{cm}^{-1}\right): 3434,1730,1696$, 1650; ${ }^{1} \mathrm{H}$ NMR $\left(\mathrm{CDCl}_{3}\right) \delta: 6.93\left(\mathrm{dq},{ }^{1} \mathrm{H}, \mathrm{J}=1.5-7.2 \mathrm{~Hz}, \mathrm{H}\right.$-tig); $5.44(\mathrm{~m}$, $\left.{ }^{1} \mathrm{H}, \mathrm{H}-6\right)$; 4.65 (dd, $\left.{ }^{1} \mathrm{H}, \mathrm{J}=4.5-11.7 \mathrm{~Hz}, \mathrm{H}-12 \alpha\right)$; 4.65-4.55 (m, $\left.{ }^{1} \mathrm{H}, \mathrm{H}-3 \alpha\right)$; 4.29 (s, $\left.{ }^{1} \mathrm{H}, \mathrm{OH}\right) ; 3.15$ (m, $\left.{ }^{1} \mathrm{H}, \mathrm{H}-17 \mathrm{\alpha}\right) ; 2.50-2.05$ (m, 3H); 2.21 (s, 3H, $\left.\mathrm{CH}_{3}, \mathrm{H}-21\right) ; 2.05-0.90(\mathrm{~m}, 13 \mathrm{H}) ; 2.03\left(\mathrm{~s}, 3 \mathrm{H}, \mathrm{CH}_{3}, \mathrm{Ac}\right) ; 1.89\left(\mathrm{~s}, 3 \mathrm{H}, \mathrm{CH}_{3}\right.$, $\mathrm{H}$-tig); 1.84 (dd, 3H, CH, J=0.9-6.3 Hz, H-tig); 1.07 (s, 3H, $\mathrm{CH}_{3}, \mathrm{H}-18$ ); $1.02\left(\mathrm{~s}, 3 \mathrm{H}, \mathrm{CH}_{3}, \mathrm{H}-19\right) .{ }^{13} \mathrm{C} \mathrm{NMR}\left(\mathrm{CDCl}_{3}\right) \delta: 217.0(\mathrm{C}=\mathrm{O}, \mathrm{C}-20) ; 170.5$ $(\mathrm{C}=\mathrm{O}, \mathrm{OAc}) ; 167.7(\mathrm{C}=\mathrm{O}, \mathrm{C}-\mathrm{tig}) ; 137.8(\mathrm{C}=\mathrm{CH}, \mathrm{C}-\mathrm{tig}) ; 123.0(\mathrm{C}=\mathrm{CH}$, C-6); 85.7 (C, C-14); 75.9 (CH, C-3); 73.9 (CH, C-12); $57.2(\mathrm{CH}, \mathrm{C}-17)$; 53.7 (C, C-13); 42.9 (CH, C-9); $37.8\left(\mathrm{CH}_{2}, \mathrm{C}-1\right) ; 36.9\left(\mathrm{CH}_{2}, \mathrm{C}-4\right) ; 36.9$ (C, C-10); $35.6\left(\mathrm{CH}_{3}, \mathrm{C}-21\right) ; 34.4\left(\mathrm{CH}_{2}, \mathrm{C}-15\right) ; 33.2(\mathrm{CH}, \mathrm{C}-8) ; 27.6$ $\left(\mathrm{CH}_{2}, \mathrm{C}-2\right) ; 27.3\left(\mathrm{CH}_{2}, \mathrm{C}-7\right) ; 26.0\left(\mathrm{CH}_{2}, \mathrm{C}-11\right) ; 24.4\left(\mathrm{CH}_{2}, \mathrm{C}-16\right) ; 21.4$ $\left(\mathrm{CH}_{3}, \mathrm{Ac}\right) ; 19.3\left(\mathrm{CH}_{3}, \mathrm{C}-19\right) ; 14.5$ (CH3, C-tig); $12.2\left(\mathrm{CH}_{3}, \mathrm{C}\right.$-tig); 9.9 (CH3, C-18). HRMS (ESI) m/z: $\mathrm{C}_{28} \mathrm{H}_{40} \mathrm{NaO}_{6}[\mathrm{M}+\mathrm{Na}]+$ calcd. 495.2717, found 495.2693, [a]20D: +5 (c 0.01, $\mathrm{CHCl}_{3}$ ).

\section{2-O- $\beta$-tigloyl-3 $\beta, 14 \beta$-dihydroxy-pregn-5-ene-20-one (Hoodigonenin A)}

A solution of compound $13(42 \mathrm{mg}, 0.09 \mathrm{mmol})$ in $\mathrm{MeOH}(5 \mathrm{~mL})$ was treated with $\mathrm{K}_{2} \mathrm{CO}_{3}(33 \mathrm{mg}, 0.24 \mathrm{mmol})$. The reaction mixture was stirred for $2 \mathrm{~h}$ at r.t. Water $(10 \mathrm{~mL})$ was added and the aqueous layer was extracted with $\mathrm{CH}_{2} \mathrm{Cl}_{2}(3 \times 10 \mathrm{~mL})$. The combined organic layers were dried over $\mathrm{Na}_{2} \mathrm{SO}_{4}$, filtered and concentrated under reduced pressure. The residue was purified by column chromatography ( $5 \mathrm{~g}$ silica gel, cyclohexane-ethyl acetate, 6:4) to afford Hoodigogenin A as a white solid (26 mg, 69\%, mp 151-153 $\left.{ }^{\circ} \mathrm{C}\right)$. IR (ATR, $\left.\mathrm{cm}^{-1}\right) 3535,34711698,1682,1651{ }^{1} \mathrm{H} \mathrm{NMR}\left(\mathrm{CDCl}_{3}\right)$ $\delta: 6.91\left(\mathrm{dq},{ }^{1} \mathrm{H}, \mathrm{J}=1.6-7.2 \mathrm{~Hz}, \mathrm{H}-\mathrm{tig}\right) ; 5.40\left(\mathrm{~m},{ }^{1} \mathrm{H}, \mathrm{H}-6\right) ; 4.63$ (dd, ${ }^{1} \mathrm{H}, \mathrm{J}=4.4-$ $12.0 \mathrm{~Hz}, \mathrm{H}-12 \alpha) ; 4.26\left(\mathrm{~s},{ }^{1} \mathrm{H}, \mathrm{OH}\right) ; 3.51$ (tt, $\left.{ }^{1} \mathrm{H}, \mathrm{J}=4.6-11.2 \mathrm{~Hz}, \mathrm{H}-3 \alpha\right) ; 3.12$ (m, $\left.{ }^{1} \mathrm{H}, \mathrm{H}-17 \mathrm{\alpha}\right) ; 2.40-2.15$ (m, 3H); 2.10 (s, 3H, $\left.\mathrm{CH}_{3}, \mathrm{H}-21\right) ; 2.10-0.90$ (m, $14 \mathrm{H}) ; 1.88$ (s, 3H, $\mathrm{CH}_{3}, \mathrm{H}$-tig); 1.83 (dd, $3 \mathrm{H}, \mathrm{CH}_{3}, \mathrm{~J}=0.8-6.8 \mathrm{~Hz}, \mathrm{H}$-tig); 1.06 (s, 3H, CH, $\mathrm{H}-18$ ); 0.99 (s, 3H, CH3, H-19). ${ }^{33} \mathrm{C}$ NMR (CDCl3) $\delta: 217.1$ (C=O, C-20); 167.7 (C=O, C-tig); $139.0(\mathrm{C}=\mathrm{C}, \mathrm{C}-5) ; 137.8$ (C=C, C-tig); $128.7(\mathrm{C}=\mathrm{CH}, \mathrm{C}-\mathrm{tig}) ; 122.0(\mathrm{C}=\mathrm{CH}, \mathrm{C}-6) ; 85.7$ (C, C-14); 75.9 (CH, C-3); 71.5 (CH, C-12); 57.2 (CH, C-17); 53.8 (C, C-13); 43.1 (CH, C-9); 42.0 $\left(\mathrm{CH}_{2}, \mathrm{C}-4\right) ; 37.2\left(\mathrm{CH}_{2}, \mathrm{C}-1\right) ; 36.9(\mathrm{C}, \mathrm{C}-10) ; 35.8(\mathrm{CH}, \mathrm{C}-8) ; 34.5\left(\mathrm{CH}_{2}\right.$, $\mathrm{C}-15) ; 33.2\left(\mathrm{CH}_{3}, \mathrm{C}-21\right) ; 31.4\left(\mathrm{CH}_{2}, \mathrm{C}-2\right) ; 27.4\left(\mathrm{CH}_{2}, \mathrm{C}-7\right) ; 26.1\left(\mathrm{CH}_{2}\right.$, C-11); $24.4\left(\mathrm{CH}_{2}, \mathrm{C}-16\right) ; 19.4\left(\mathrm{CH}_{3}, \mathrm{C}-19\right) ; 14.5\left(\mathrm{CH}_{3}, \mathrm{C}-\mathrm{tig}\right) ; 12.2\left(\mathrm{CH}_{3}\right.$, C-tig); $9.9\left(\mathrm{CH}_{3}, \mathrm{C}-18\right)$. HRMS (ESI) m/z: $\mathrm{C}_{26} \mathrm{H}_{38} \mathrm{NaO}_{5}[\mathrm{M}+\mathrm{Na}]+$ calcd. 453.2611, found 453.2576, [a]20D: +1.13 (c 0.006, $\mathrm{CHCl}_{3}$ ).

\section{References}

1. World Health Organization.

2. van Heerden FR, Marthinus Horak R, Maharaj VJ, Vleggaar R, Senabe JV, et al. (2007) An appetite suppressant from Hoodia species. Phytochemistry 68 2545-2553.

3. van Heerden FR (2008) Hoodia gordonii: a natural appetite suppressant. J Ethnopharmacol 119: 434-437.

4. Shukla YJ, Pawar RS, Ding Y, Li X-C, Ferreira D, et al. (2009) Pregnane glycosides from Hoodia gordonii. Phytochemistry 70: 675-683.

5. Pawar RS, Shukla YJ, Khan SI, Avula B, Khan IA (2007) New oxypregnane glycosides from appetite suppressant herbal supplement Hoodia gordonii Steroids 72: 524-534.

6. Just IG, Engel ChR (1958) Steroids and related products. IX. Introduction of type $\Delta^{11}$-double bond. J. Org. Chem 23: 12-15

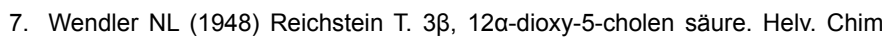
Acta 31: 1713-1719.

8. Villoti R, Djerassi C, Ringold HL (1959) Steroids. CXXIII. 19-nor-6methylandrostane derivatives. J. Am. Chem. Soc 81: 4566-4570.

9. Jiang X, Covey DF (2002) Total synthesis of ent-cholesterol via a steroid C D-ring side-chain synthon. J Org Chem 67: 4893-4900.

10. Dess DB, Martin JC (1983) Readily accessible 12-I-5 oxidant for the conversion of primary and secondary alcohols to aldehydes and ketones. J Org Chem 48 : 4155-4156.

11. Welzel P, Janssen B, Duddeck H (1981) $14 \beta$-Hydroxy steroids. II. Prins reaction of lumihecogenin acetate. Liebigs Ann Chem 546-64.

12. Welzel P, Stein H (1981) 14ß-Hydroxy steroids. III. Synthesis of digoxigenin from deoxycholic acid. Tetrahedron Lett 22: 3385-8.

13. Milkova T, Stein H, Ponty A, Boettger D, Welzel P (1982) $14 \beta$-Hydroxy steroids. VI. Synthesis of digitoxigenin. Tetrahedron Lett 23: 413-14.

14. Welzel P, Moschner R, Ponty A, Pommerenk U, Sengewein H (1982) $14 \beta$-Hydroxysteroids. V. Synthesis of digipurpurogenin I and II derivatives. Liebigs Ann Chem 564-78.

15. Welzel P, Stein H, Milkova T (1982) 14ß-Hydroxy steroids. VII. Synthesis of digoxigenin and digitoxigenin from deoxycholic acid. Liebigs Ann Chem 2119 34.

16. LaCour TG, Guo C, Bhandaru S, Fuchs PL, Boyd MR (1998) Interphylal Product Splicing: The First Total Syntheses of Cephalostatin 1, the North Hemisphere of Ritterazine G, and the Highly Active Hybrid Analog, Ritterostatin GN1N. J Am Chem Soc 12: 692-707 4

17. Geoffroy $P$, Ressault B, Marchioni E, Miesch M (2011) Norrish-Prins reaction as a key step in the synthesis of $14 \beta$-hydroxy- $5 \mathrm{Ha}$ (or $5 \mathrm{H} \Delta$ or $\Delta^{5,6}$ )-pregnane derivatives Steroids 76: 1166-1175

18. Geoffroy P, Ressault B, Marchioni E, Miesch M (2011) Synthesis of Hoodigogenin A, aglycone of natural appetite suppressant glycosteroids extracted from Hoodia gordonii. Steroids 76: 702-708.

19. Smith C, Krysman AJ (2014) Ethnopharmacol 155: 987-991. 